\title{
A NEW APPROACH TO THE ELASTO-PLASTIC LATERAL BUCKLING STRENGTH OF BEAMS
}

\author{
By Yoshiji NIWA*, Eiichi WATANABE** and Satsuki SUZUKR***
}

\begin{abstract}
This paper is concerned with an interpretation and analysis on the elasto-plastic lateral torsional buckling of beams in a simplified manner by means of catastrophe theory. The numerical analysis does not require having recourse to the ordinary nonlinear numerical procedures, but can be performed using a relatively small microcomputer.

The proposed analysis takes into account the elasto-plastic behavior of the material, the geometric imperfections and the residual stresses. The ultimate strength of the beams is shown to be presented in the form of the bifurcation set in terms of the elastoplastic buckling moment and the imperfection term that follows the $1 / 2$ power law.

It is found from the study that the characteristics of the elasto-plastic lateral torsional buckling may be identified as the fold catastrophe just like the case of centrally compressed columns.
\end{abstract}

\section{INTRODUCTION}

Beams constitute one of the most important civil enineering structures. Slender beams under bending in the plane of the maximum flexural rigidity can become unstable by the combination of twist and lateral deflection, This buckling phenomenon is usually referred to as the lateral torsional buckling. Beams with low torsional rigidity such as of narrow rectangular section or I-section are known to be vulnerable to this phenomenon unless they are laterally supported.

Since prandtl's initiative study on the lateral elastic buckling in 1899, a great number of researches have been underway including the elasto-plastic buckling. The early developments of the study have been surveyed by G. C. Lee in his literature survey ${ }^{11}$. A method of summarizing a great number of available theoretical solutions in a useful way has been suggested by Nethercot and Rockey"). The detailed discussions on the basic concepts underlying the lateral buckling and on the design methods in various countries are provided in a text book by Allen and Bulson ${ }^{2)}$. The current interests in the design of laterally unsupported beams are now focused on their elasto-plastic strength taking into account such effects as the support conditions, the shape of bending moment distribution, the position of load application, the shape of cross section, the residual stresses and geometrical imperfections ${ }^{3)}$.

In this paper, however, the main interest of the study is focused on the application of the catastrophe theory in the elasto-plastic range. Thus, only the simplest models of beams are considered herein.

\section{BASIC ASSUMPTIONS}

The basic assumptions made herein are summarized as follows :

* M. JSCE. Dr. Eng. . Professor of Kyoto University (Sakyo-Ku, Kyoto 606)

** M. JSCE, Ph. D. . Associate Professor of Kyoto University (Sakyo-Ku, Kyoto 606)

**:* M. JSCE, M. S. . Engineer, Aichi Prefectural Government (Okazaki, Aichi 444) 
(1) The beams are of doubly symmetric cross section and are subjected to the pure bending moment, $M_{x}$, acting in the plane of the web as shown in Fig. 1 :

where

$b$ : width of the flange plate,

$h:$ depth of the web,

$t_{f}:$ thickness of the flange plate,

$t_{w}:$ thickness of the web,

$l$ : lateral buckling length,

$u$ : lateral deflection,

$v$ : vertical deflection,

$\phi$ : angle of rotation.

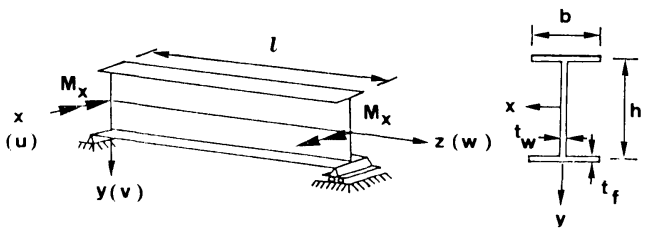

Fig. 1. Beams under pure moment.

(2) Both ends are simply supported, namely,

$u=u^{\prime \prime}=\phi=\phi^{\prime \prime}=0$ at $z=0$ and $z=l$.

where the prime designates the differentiation with respect to $z$.

(3) The material is ideally perfectly elasto-plastic as is shown in

Fig. 2 :

(4) Residual stress is taken into account only in the flange plates.

It is assumed to be distributed in the triangular form as shown in

Fig. 3. where $\sigma_{r}$ refers to the magnitude of the residual stress.

(5) The cross section does not undergo distortion.

(6) Neither the gravity center nor the shear center changes the original position.

(7) The plastic portion of the cross section is assumed to provide no resistance to further bending.

(8) The rotation and the geometric imperfection are assumed only in the following form :

stress

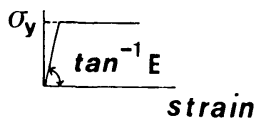

Fig. 2. Perfectly elasto-plastic material.

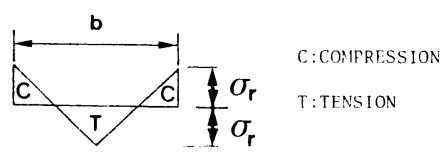

Fig. 3. Triangular residual stress in flange plate.

$$
\phi=\theta \sin \frac{\pi z}{l}, \phi_{0}=\theta_{0} \sin \frac{\pi z}{l} \text {. }
$$

(9) The vertical deflection $v$ is assumed to be small.

\section{(1) Elastic Buckling}

Before discussing the elasto-plastic buckling, let us briefly summarize the classical elastic buckling problem. If the global and local coordinate system is designated respectively by $(x, y, z)$ and $(\xi, \eta, \zeta)$, then the small deformations of the beam in $x$ - and $y$ - direction are sketched as in Fig. 4 :

Since the direction cosines are related as shown in Table 1, each component of the applied bending moment in the local coordinate system is given respectively by :

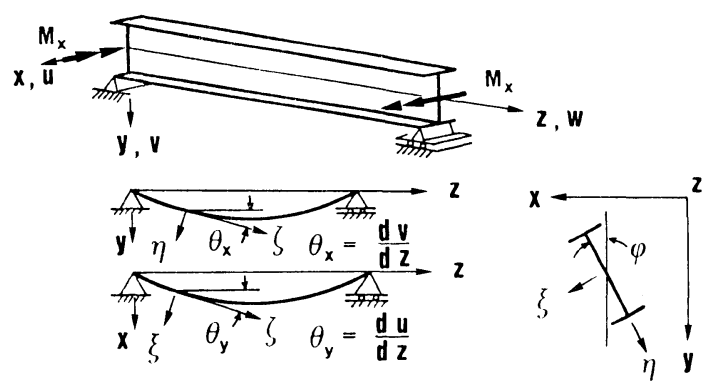

Fig. 4. Configuration of laterally buckled beam.

$$
\left.\begin{array}{l}
M_{\xi}=M_{x} \\
M_{\eta}=\phi M_{x} \\
M_{5}=M_{x} \frac{d u}{d z}
\end{array}\right\}
$$

Table 1. Direction cosines.

\begin{tabular}{|c|c|c|c|}
\hline$y$ & $x$ & $y$ & $z$ \\
\hline 5 & 1 & $\phi$ & $-\frac{d u}{d z}$ \\
\hline$n$ & $-\phi$ & 1 & $-\frac{d v}{d z}$ \\
\hline$\zeta$ & $\frac{d u}{d z}$ & $\frac{d v}{d z}$ & 1 \\
\hline
\end{tabular}

Then, the basic equations of equilibrium can be obtained in the following:

$\left.\begin{array}{l}M_{x} \phi=-E I_{y e} u^{\prime \prime} \quad \text { with respect to } \eta \text {-axis } \\ M_{x}=-E I_{x e} v^{\prime \prime} \quad \text { with respect to } \xi \text {-axis } \\ M_{x} u^{\prime}=G J \phi_{e}^{\prime}-E C_{w e} \phi_{e}^{\prime \prime \prime} \quad \text { with respect to } \zeta \text {-axis, }\end{array}\right\}$ 
where

$I_{y e}:$ moment of inertia about $y$-axis,

$I_{x e}$ : moment of inertia about $x$-axis,

$G J:$ St. Venant torsional rigidity

$E C_{w e}$ : warping rigidity

$\phi_{e}=\phi-\phi_{0}$

The substitution of the 3rd equation of Eq. (4) into the lst equation yields the following well-known equation :

$G J \phi_{e}^{\prime \prime}-E C_{w e} \phi_{e}^{\prime \prime \prime \prime}=-\frac{M_{x}^{2}}{E I_{y e}} \phi$

The case of plastic buckling has been treated by Kachanovi).5) within the framework of the theory of plasticity making a modification to the elastic moduli*. One may assume that flexural and torsional rigidities are reduced in accordance with the tangent modulus theory like Flint ${ }^{(6)}$. The more accurate analyses for I-beams have been proposed by Nethercot ${ }^{7)}$ and Galambos ${ }^{8)}$ in such a fashion that a perfect elasto-plasticity is assumed and the torsional rigidity remains at its elastic value; whereas, the flexural and warping rigidity are reduced by the tangent modulus factor. It is concluded that the lateral buckling strength, $\boldsymbol{M}_{\boldsymbol{c} r}$, can be reduced by as low as $30 \%$ for some range of the slenderness ratio if the residual stress is present.

\section{( 2 ) Elasto-Plastic Lateral Buckling}

The proposed analysis is based on the modification of the elastic equations ${ }^{9)}{ }^{11)}$. The residual stress distibution considered herein is of the triangular form in the flanges and the stress distribution will become as shown in Fig. 5 under the applied bending moment, $M_{x}$ :

Following Galambos ${ }^{8)}$ and Nethercot ${ }^{7)}$, a modification may be made to the flexural and warping rigidities in the subsequent manner:

$$
\left.\begin{array}{l}
E I_{y}=k_{1} E I_{y e} ; \quad k_{1}=\frac{1}{2}\left[k^{3}+k^{\prime}\left(3-3 k^{\prime}+k^{\prime 2}\right)\right] \\
E C_{w}=k_{2} E C_{w e} ; \quad k_{2}=\frac{2 k^{3} k^{\prime}\left(3-3 k^{\prime}+k^{\prime 2}\right)}{k^{3}+k^{\prime}\left(3-3 k^{\prime}+k^{\prime 2}\right)}
\end{array}\right\}
$$

where $k$ and $k^{\prime}$ refers to the ratio of the elastic portion of the compression and the tension flange, respectively, to the total width of the cross section.

Thus, the equation of equilibrium, originally given by $\mathrm{Eq}(6)$ can be modified in the following form : **

$$
k_{1} E I_{y} G J \phi_{e}^{\prime \prime}-k_{3} E I_{y} E C_{w} \phi_{e}^{\prime \prime \prime \prime}=-\phi M_{x}^{2}
$$

where

$$
\phi_{e}=\phi-\phi_{0}
$$

$\phi_{0}$ : initial twist, namely, the imperfection

$k_{3}=k_{1} k_{2}=k^{\prime} k^{3}\left(3-3 k^{\prime}+k^{\prime 2}\right)$

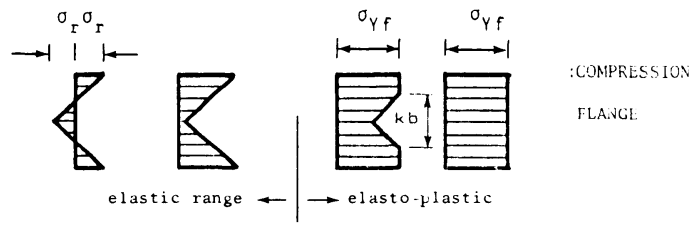

(a)

Now, let us assume the solution $\phi$ and the imperfection $\phi_{0}$ in the following form, respectively:

$$
\phi=\theta \sin \frac{\pi z}{l} ; \quad \phi_{0}=\theta_{0} \sin \frac{\pi z}{l}
$$

Then, the equation of equilibrium can be given by :

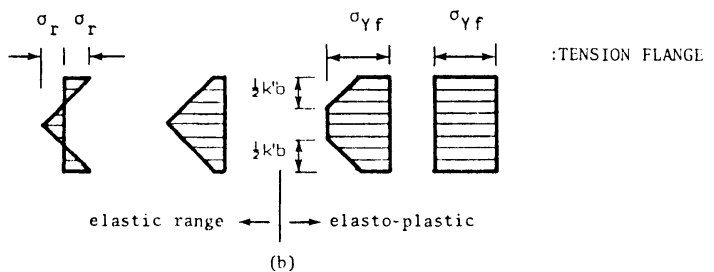

Fig. 5. Triangular residual stress distribution in flanges.

* Kachanov treats inelastic lateral bucklings of beams under pure bending and cantilevered beams under concentrated load at the free end The effect of residual stresses is not considered.

**Hereafter, subscript $e$ on the rigidities will be omitted for simplicity. Furthermore, it is particularly to be noted that coefficients $k_{1} \& k_{3}$ are evaluated assuming the ideally perfect beams without the imperfection.

The effect of the imperfection upon $k_{1} \& k_{3}$ thus is not explicitly taken into account but may be so implicitly by means of the equivalent imperfection that is defined afterwards. 


$$
\begin{aligned}
& \quad\left[\left\{k_{1} G J E I_{y}\left(\begin{array}{c}
\pi \\
l
\end{array}\right)^{2}+k_{3} E C_{w} E I_{y}\left(\begin{array}{c}
\pi \\
l
\end{array}\right)^{4}\right\} \theta_{e}-M_{x}^{2} \theta\right] \sin \frac{\pi z}{l}=0 \\
& \text { where } \\
& \quad \theta_{e}=\theta-\theta_{0}
\end{aligned}
$$

The equation of equilibrium may be rewritten so that the problem is treated as if it were nonlinear elastic and the first partial derivative of a certain pseudo-potential $U$ with respect to the generalized coordinate $\theta$ vanishes $\left.:{ }^{9)} \sim 11\right)$

$$
U_{1} \equiv \frac{\partial U}{\partial \theta}=f E C_{w} E I_{y}\left(\begin{array}{c}
\pi \\
l
\end{array}\right)^{4} \theta_{e}-M_{x}^{2} \theta=0
$$

where $f$ indicates a secant factor $f=f\left(\theta_{e}\right)$ such that

$$
\left.\begin{array}{l}
f^{c} \equiv f(0)=k_{4}=k_{3}\left(1+k_{1} \eta\right) \\
\text { where } \\
\eta \equiv\left(\frac{l}{\pi}\right)^{2} \frac{G J}{E C_{w}}
\end{array}\right\}
$$

Following the procedure adopted in the analysis of elasto-plastic buckling of columns, the secant factor $f\left(\theta_{e}\right)$ can be evaluated in the form : ${ }^{9}$

$$
\begin{gathered}
f\left(\theta_{e}\right)=f^{c}+\frac{1}{2} f_{t}^{\prime c} \theta_{e} \\
\text { where } \\
f_{t}^{\prime c} \equiv \frac{\partial f}{\partial \theta_{e}}
\end{gathered}
$$

Let the surface of the equilibrium be designated by $M_{U}$ and let it be expressed in terms of the coordinates $\left(\theta, \theta_{0}\right.$, $M_{x}^{2}$ ), then it can be graphically drawn as shown in Fig. 6 . The solid curve $A C E$ or $A C F$ corresponds to the perfect system, $\theta_{0}=0$. Moreover. the critical pathological curve can be obtained in such a way that the mapping from a set of coordinates $\left(\theta, \theta_{0}\right)$ to that of $\left(\theta_{0}, M_{x}^{2}\right)$ through equilibrium surface $M_{U}$ becomes singular. This is equivalent to say that the Jacobian $J\left(\theta_{0}, M_{x}^{2} ; \theta, \theta_{0}\right)$ vanishes or the second partial derivative of $U\left(\theta, \theta_{0}, M_{x}^{2}\right)$ with respect to $\theta$ vanishes.

It has been demonstrated in reference 9) that the stability of the system can be discussed by evaluating the partial derivatives of the pseudo-potential such as shown subsequently :

$$
\begin{aligned}
& U_{11} \equiv \frac{\partial^{2} U}{\partial \theta^{2}}=\left(f^{c}+f_{t}^{\prime c} \theta_{e}\right)\left(\frac{\pi}{l}\right)^{4} E C_{w} E I_{y}-M_{x}^{2} \\
& \dot{U}_{1} \equiv \frac{\partial U_{1}}{\partial \theta_{0}}=-f E C_{w} E I_{y}\left(-\frac{\pi}{l}\right)^{4} \\
& \stackrel{\circ}{U}_{11} \equiv \frac{\partial U_{11}}{\partial\left(M_{x}^{2}\right)}=-1 \\
& U_{111} \equiv \frac{\partial^{3} U}{\partial \theta^{3}}=f_{t}^{\prime c} E C_{w} E I_{y}\left(\frac{\pi}{l}\right)^{4} \neq 0^{*}
\end{aligned}
$$
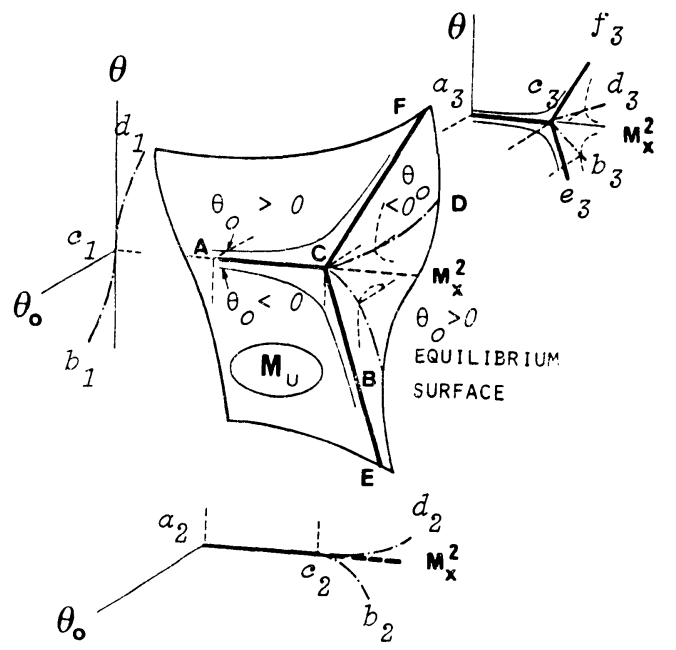

Fig. 6. Equilibrium surface $M_{U}$.

For the perfect beam free from the imperfection, it follows that

$$
M_{x}^{2}=\left(f^{c}+f_{t}^{\prime c} \theta\right) E C_{w} E I_{y}\left(\frac{\pi}{l}\right)^{4}
$$

thus, the elasto-plastic buckling momen: $M_{x c r}$ can be obtained as :

$$
M_{x c r}^{2}=f^{c} E C_{w} E I_{y}\left(\frac{\pi}{l}\right)^{4}
$$

\footnotetext{
*Thus, the catastrophe is identified to be "fold", that is, "asymmetric bifurcation buckling"
} 
Consequently, the pathological critical curve of $M_{x}-\theta$ can be shown to be given by :

$$
\left(\frac{M_{x}}{M_{x c r}}\right)^{2}=1-\alpha \theta
$$

where

$$
\alpha \equiv-\frac{f_{i}^{\prime c}}{f^{c}}
$$

Due to the Shanley effect, it can be shown that $\alpha<0^{9)}$. Thus, Eq. (17) designates straight lines $C E$ and $C F$ in Fig. 6. Consequently, the buckling point and its vicinity are characterized by the stable symmetric buckling in a global sense. This implies that there exists no extremum value of $M_{x}^{2}$ around here and the evaluation of the imperfection sensitivity must be performed at some point other than this buckling point*

The elasto-plastic buckling moment can be computed from Eq. (16) by letting $f^{c}=k_{4}$. For simplicity. let us assume that the residual stress in the web is self-balanced and the in-plane bending strain of the web is equal to that of the flange at the junction to each flange and that the effect of the residual stress in the web upon the yielding of the web is negligible. Then, the stress distribution in the web can be seen to be symmetric with respect to the $x-x$ axis and $M_{x c r}$ can be obtained in a reasonably approximate manner with the aid of Fig. 7 , and using the average stresses in the flange plates $\tilde{\sigma}=\left(1-k^{2} \tilde{\sigma}_{r}\right)$ and the web bending stress $\tilde{\sigma}_{w u}=1+\tilde{\sigma}_{r}(1-2 k)$ at the junction of the web and each flange by the following equation :

$$
\begin{aligned}
& \frac{\sqrt{k_{\ell}}}{h A_{f} \sigma_{\gamma_{f}}}\left(\frac{\pi}{l}\right)^{2} \sqrt{E C_{w} E I_{y}}=1-k^{2} \tilde{\sigma}_{r}+\frac{\sigma_{Y w} A_{w} /\left(A_{f} \sigma_{Y S}\right)}{\left[1+\tilde{\sigma}_{r}(1-2 k)\right]^{2}}\left\{\frac{\tilde{\sigma}_{r}}{4}(1-2 k)\right. \\
& \left.\left[2+\tilde{\sigma}_{r}(1-2 k)\right]+\frac{1}{6}\right) \text { for } 0<k \leqq \frac{1}{2} \zeta \\
& =1-k^{2} \tilde{\sigma}_{r}+\frac{1}{6}\left[1+\tilde{\sigma}_{r}(1-2 k)\right] \frac{A_{w}}{A_{\zeta}} \text { for } 1 \geqq k>\frac{1}{2} \zeta \mid \zeta \equiv \frac{1}{\sigma_{r}}\left(1-\frac{\sigma_{\gamma w}}{\sigma_{Y, f}}\right)+1
\end{aligned}
$$

Again, following the same procedure as for the column, let us consider the "equivalent bifurcation point" which is obtained as the intersection of the postbucking path $A C$ and the curve of failure mechanism $B C D$ in Fig. $8^{9)}$.

In order to obtain the failure mechnism curve, the interaction curve and the moment-rotation relationship will be necessary:

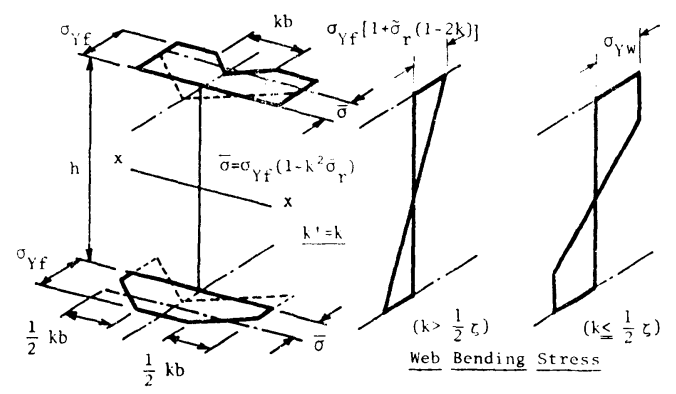

Fig. 7. Evaluation of elasto-plastic buckling of beams.

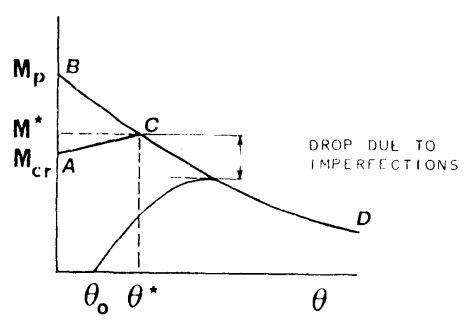

Fig. 8. Equivalent bifurcation point for beam.

$$
m_{x}^{2}+m_{y}=1: \text { interaction curve } \mathbf{e}^{12)}
$$

where

$$
\begin{aligned}
& m_{x}=\frac{M_{x}}{M_{x p}} ; \quad M_{x p}=\text { plastic moment }(x \text {-axis } \\
& m_{y}=\frac{M_{y}}{M_{y p}} ; \quad M_{y p}=\text { plastic moment } @ y \text {-axis }
\end{aligned}
$$

and

\footnotetext{
*e. g. Point $C$ in Fig. 8.

${ }^{* *} \bar{\sigma} \equiv \sigma / \sigma_{Y f}:$ non-dimensionalized stress in terms of the yield stress
} 
$M_{y}=\phi M_{x}:$ moment-rotation relationship, Eq. (3)

As far as the beams are concerned, the postbuckling reservation may be thought to be quite insignificant so that path $A C$ will be assumed to be a horizontal line in the subsequent analysis.

Upon substitution of Eq. (19) into Eq. (20), the following equation can be obtained :

$m_{y}=\tau m_{x} \theta=1-m_{x}^{2}$

where

$$
\tau=\frac{M_{x \rho}}{M_{y \rho}}
$$

Thus, the generalized angle of rotation, $\theta$, can be expressed as :

$$
\theta=\frac{1}{\tau}\left(\frac{1}{m_{x}}-m_{x}\right)
$$

This relationship can be designated by Curve $B C D$ in Fig. 8 , and represents a pathological limit in a sense that the strength of beams can never exceed the value obtained by Eq. (22). From this consideration, the value of $\alpha$ in Eq. (17) is assumed to be approximated by the slope of Curve $B C D$ :

$$
\alpha=-\frac{d}{d \theta}\left(\frac{\boldsymbol{M}_{x}^{2}}{\boldsymbol{M}_{x c r}^{2}}\right)=2 \frac{m_{x}}{1+m_{x}^{2} \tau}
$$

\section{(3) Equivalent Imperfection and Imperfection Sensitivity}

The strength of actual beams is affected by the imperfection as well as by the residual stress, and is control'ed by the plastic unloading curve. Thus, the following arguments are provided with a view to utilizing the intersection point $C$ in Fig. 8 as the equivalent bifurcation point in stead of the original bifurcation point $A$.

Since the relationship between the torque and the rate of twist is assumed to take form invariant to the magnitude of $\theta_{0}$, it can be shown that the equation of equilibrium can be rewritten using another pseudo-potential $V\left(\theta-\theta^{*}, \theta_{0}\right.$, $\left.M_{x}^{2}\right)$ about the "equivalent bifurcation point", Point $C$ in Fig. 8 :

$$
V^{\prime}=M_{E}^{2} f\left(\theta_{d}^{*}\right) \theta_{d}^{*}-M_{x}^{2}\left(\theta-\theta^{*}\right)=0
$$

where

$$
\begin{aligned}
& f\left(\theta_{d}^{*}\right)=f^{c}+\frac{1}{2} f_{t}^{\prime c} \theta_{d}^{*} \\
& \theta_{d}^{*}=\theta-\theta^{*}-\theta_{0} \\
& M_{E}=\left(\frac{\pi}{l}\right)^{2} \sqrt{E C_{w} E I_{y}} \text { : Euler buckling moment }
\end{aligned}
$$

Fig. 9 shows the surface of the equilibrium, $M_{v}$, expressed in terms of the coordinate system $\left(\theta-\theta^{*}, \theta_{0}, M_{x}^{2}\right)$. In order to obtain the relationship between the maximum bending moment, $m_{x u}$, and imperfection parameter, $\theta_{0}$, one may use a criterion that the transform from a pair of coordinate system $\left(\theta-\theta^{*}, \theta_{0}\right)$ to the pair of coordinate system $\left(\theta_{0}, M_{x}^{2}\right)$ through $M_{V}$ becomes singular. Or, one may evaluate the 2 nd partial derivative of $V$ with respect to $\theta$ and let it vanish.

The relationship between $M_{x}$ and $\theta_{0}$ obtained through this singularity transform is usually referred to as the bifurcation set. or in other words, the imperfection sensitivity curve. of the beam. It can be expressed in the following form :

$$
m_{r u}=m_{x c r} \sqrt{1+\alpha \theta_{0}-\sqrt{2 \alpha \theta_{0}\left(1+\frac{\alpha \theta_{0}}{2}\right)}}
$$

The cross sections are conveniently assumed to be

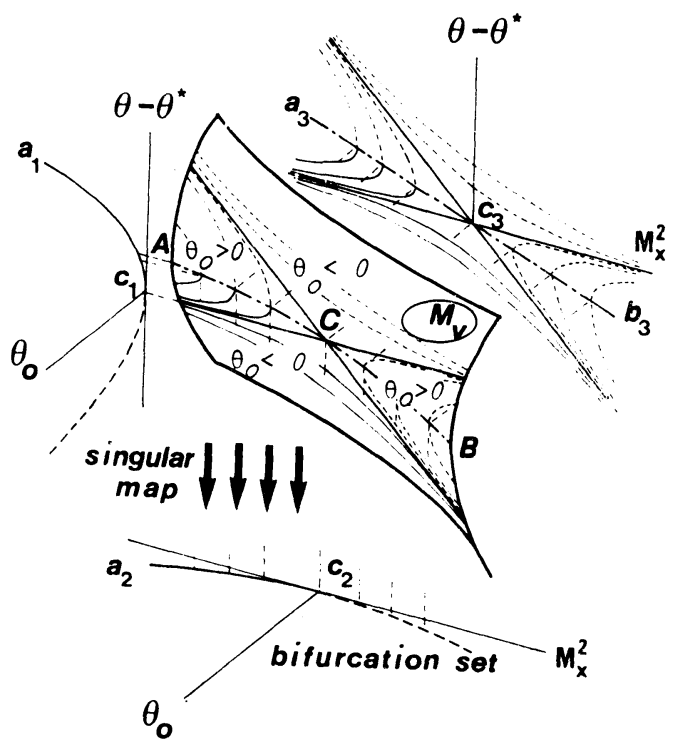

Fig. 9. Equilibrium space $M_{v}$ and singular bifurcation set. 
doubly symmetric as shown in Fig. 1. The section properties thus can be given by :

$$
\begin{aligned}
& I_{y}=\frac{1}{6} A_{f} b^{2} \\
& I_{x}=\frac{A_{f} h^{2}}{2}\left(1+\frac{A_{w}}{6 A_{f}}\right) \\
& C_{w}=\frac{I_{y}}{4} h^{2}=\frac{A_{f}}{24} b^{2} h^{2} \\
& J=\frac{2}{3} A_{f} t_{f}^{2}\left\{1+\frac{A_{w}}{2 A_{f}}\left(\frac{t_{w}}{t_{f}}\right)^{2}\right\}
\end{aligned}
$$

Then, the elasto-plastic lateral torsional buckling moment, $m_{x c r}$, can be expressed by the following formula :

$$
m_{x c r}=\sqrt{f^{c}} \frac{\gamma}{\lambda^{2}}
$$

where

$$
\begin{gathered}
\lambda=\frac{2 l}{\pi b} \sqrt{3\left(1+\frac{A_{w}}{6 A_{f}}\right)^{\sigma_{Y S}}} E \\
\text { and } \gamma=\frac{1+\begin{array}{c}
A_{w} \\
6 A_{s}
\end{array}}{1+A_{w} \sigma_{Y w}} \\
4 A_{f} \sigma_{Y S}
\end{gathered}
$$

where $\lambda$ is referred to as the generalized slenderness ratio for the lateral torsional buckling of beams

Following reference 9), the imperfection $\theta_{0}$ may be replaced conveniently by the same expression of the equivalent imperfection $\theta_{0}^{*}$ that has been introduced in the case of columns:

$$
\left.\begin{array}{l}
\theta_{0}^{*} \equiv \mu(\lambda) \theta_{0} \\
\mu(\lambda) \equiv \begin{array}{l}
1 \\
2
\end{array}\left(\begin{array}{c}
\lambda \\
\lambda_{p}
\end{array}\right)^{\nu}, \quad \nu \equiv 2\left(1-\frac{\lambda}{\lambda_{p}}\right)
\end{array}\right\}
$$

where $\lambda_{p}$ refers to the value of $\lambda$ at which the elasto-plastic buckling curve bifurcates from the Euler curve. This is to take into account of the fact that neither very stocky nor very slender beam is sensitive to the imperfection. The detailed discussion on this matter is provided in two references ${ }^{9)}{ }^{11}$ and is not repeated here due to the limitation of pages.

Figs. 10 to 13 show several examples of the numerical analysis on the strength of laterally unsupported I -beams with certain combinations of parameters: $A_{w} / A_{f}, h / b, A_{f} /(b h), \theta_{0}, \sigma_{r}$. and $(l / \pi)^{2} G J /\left(E C_{w}\right)$. Shown are (a) the elasto-plastic buckling curve, (b) the bifurcation set, (c) the JRA basic beam strength curve $^{(3)}$. (d) the beam curve by Trahair ${ }^{14)}$. and (e) the ECCS curve* for $n=1.5$ with some experimental values of beams either rolled or welded quoted from references ${ }^{14)}{ }^{15)}$. It is to be noted. however, that curves (c) and (d) are

${ }^{*}$ ECCS CURVE : $M / M_{\rho}=1 /\left(1+\lambda^{2 n}\right)^{1 / n}$

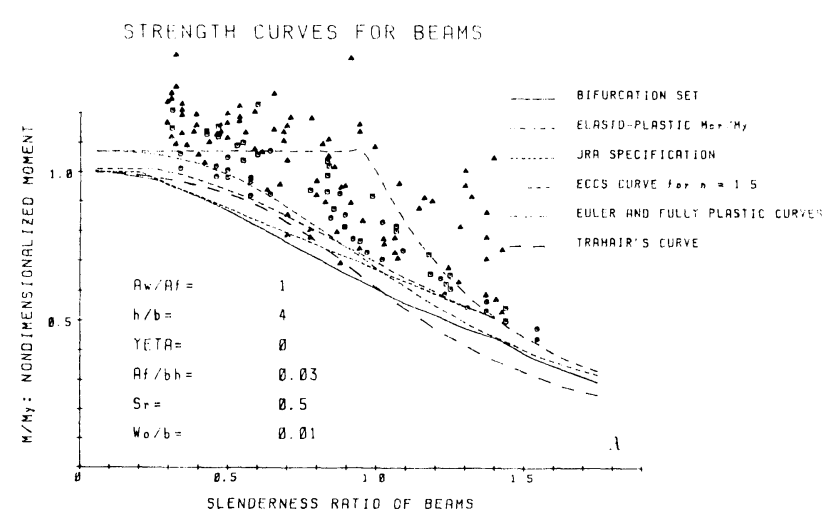

Fig. 10. Beam strength curves $\left(A_{w} / A_{f}=1, h / b=4, \eta=0\right.$ $A_{s} /(b h)=0.03, \quad \tilde{\sigma}_{r}=0.5$ and $\left.\theta_{0}=0.01\right)$

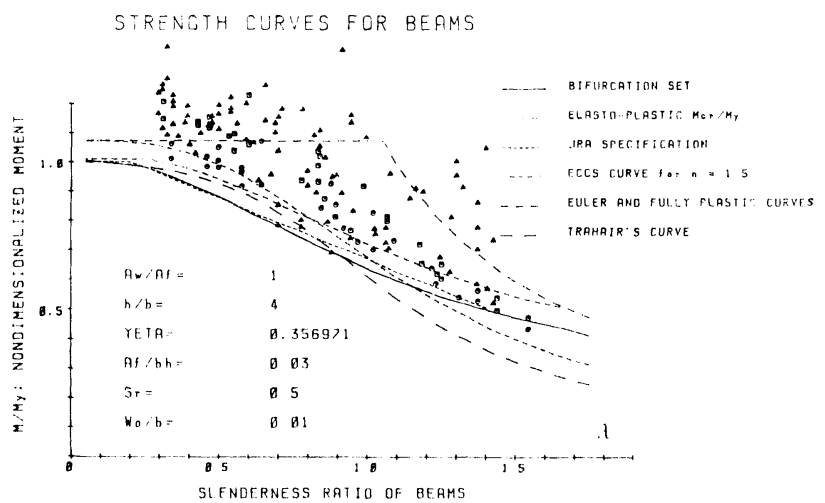

Fig. 11. Beam strength curves $\left(A_{w} / A_{f}=1, h / b=4, \eta=0.36\right.$. $A_{f} /(b h)=003 . \quad \tilde{\sigma}_{r}=0.5$ and $\left.\theta_{0}=0.01\right)$ 


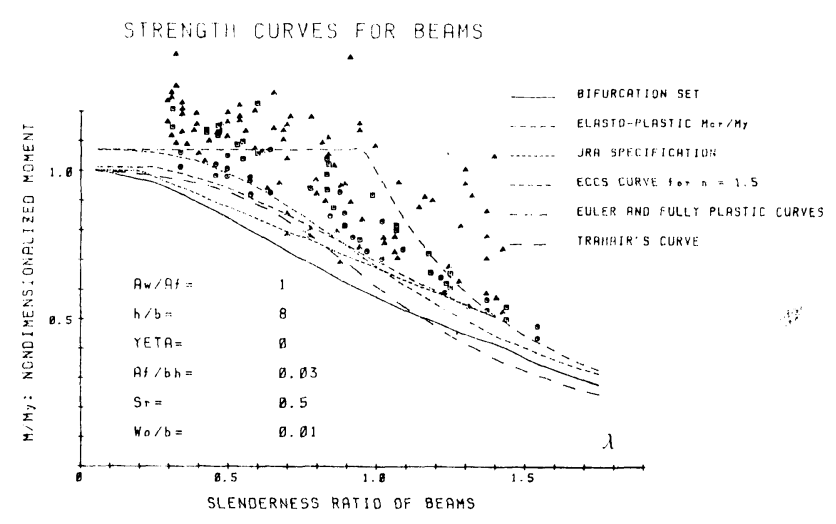

Fig. 12. Beam strength curves $\left(A_{w} / A_{f}=1, h / b=8, \eta=0\right.$, $A_{,} /(b h)=0.03, \quad \bar{\sigma}_{r}=0.5$ and $\left.\theta_{0}=0.01\right)$

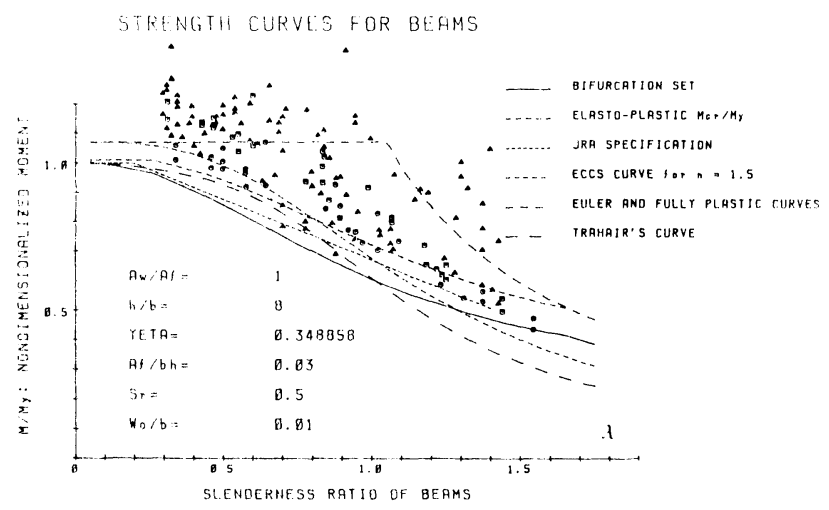

Fig. 13. Beam strength curves $\left(A_{w} / A_{s}=1, h / b=8, \eta=0.35\right.$. $A_{s} /(b h)=0.03, \quad \tilde{\sigma}_{r}=0.5$ and $\left.\theta_{0}=0.01\right)$. non-dimensionalized in terms of the yielding moment ; whereas, curves (a), (b), and (e) are in terms of the fully plastic moment. The magnitude of residual stresses, $\sigma_{r}$. is assumed conservatively to be $50 \%$ of $\sigma_{Y}$ and that of the imperfection, $\theta_{0}$, is assmed also conservatively to be $1 / 100$.

Both of Fig. 10, neglecting the effect of $G J$. and Fig. 11, considering this effect, show an identical case of $A_{w} / A_{f}=1, h / b=4, A_{s} /(b h)$ $=0.03$. Whereas, Figs. 12 and 13 show another identical case of $A_{w} / A_{f}=1, h / b=8$ and $A_{f} /$ $(b h)=0.03$.

The obtained results by the proposed bifurcation set may be thought to well explain the lower bounds of the tested results, although the consideration was restricted to the simplest problem of lateral torsional buckling of beams.

\section{CONCLUSIONS}

In this paper, a new simplified approach to predict the lateral torsional buckling strength of laterally unsupported beams is proposed using concepts of the catastrophe theory. It takes into account the elasto-plasticity of the material, the residual stresses and the geometric imperfections. The proposed method is most characterized by the part of the imperfection sensitivity

function invariant to the imperfection and determined entirely on the basis of the perfect system without the initial imperfections. The main conclusions obtained through the study include the following:

(1) The elasto-plastic lateral torsional buckling of beams may be basically interpreted to be the fold catastrophe.

(2) The lateral torsional buckling strength of beams may be evaluated in terms of the bifurcation set in the catastrophe terminology and thus can be characterized by the $1 / 2$-power rule.

(3) The lower bounds of the tested results of beams quoted here can be well predicted by the proposed bifurcation set.

This study was financially assisted by a Grant-in-Aid for Scientific Research from the Ministry of Education. Science and Culture in the years of 1981-1982. The numerical computations were mainly performed using a relatively small microcomputer.

\section{REFFERENCES}

1) Lee. G. C. : A survey of literature on the lateral instability of beams, Welding Research Council Bulletin, No 63, 1960.

2) Allen, H. G. and Bulson, P. S. : Background to buckling, McGraw-Hill Book Co. , 1980

3) Nethercot, D. A. Design of beams and plate girders-Treatment of overall and local flange buckling. Design of Steel Structures, Paper 13, Granada, 1982.

4) Kachanov, L. M. : Foundations of the theory of plasticity. Series in Applied Mathematics and Mechanics, North-Holland Publishing Company, Amsterdam, 1971.

5) Kachanov. L. M. : A brief course on the theory of buckling. Solid Mechanics Division. University of Waterloo Press. Canada, 1981 
6) Flint, A. R. : Stability and strength of stocky beams, Journal of Mechanics and Physics of Solids, 1.90, 1953.

7) Nethercot, D. A. : Factors affecting the buckling stability of partially plastic beams, Proc. Institution of Civil Engineers, 53. 285,1972 .

8) Galambos, T. V. : Inelastic lateral buckling of beams, Journal of the Structural Div. ASCE. ST 5, 217, 1963.

9) Niwa Y.. Watanabe, E. and Isami, H. : A new approach to predict the strength of steel columns, Proc. of JSCE, No. 341. 1984.

10) Niwa, Y., Watanabe, E. and Fukumori, Y. : On the catastrophe characteristics of elasto-plastic buckling of columns and plates (in Japanese). Theoretical and Applied Mechanics, Vol. 31, B 12, 1981, pp. 215-216.

11) Niwa, Y.. Watanabe, E. Isami H. and Fukumori, Y. : A new approach to predict the strength of compressed steel plates, Proc. of JSCE, No. 341, 1984.

12) Horne, M. R. and Morris, L. J. : Plastic design of low-rise frames, Constrado Monographs, Granada, 1981.

13) Japan Road Association, Specifications for Highway Bridges, 1980.

14) Trahair, N. S. : The behaviour and design of steel structures, Chapman and Hall, 1977.

15) Fukumoto, Y. : Numerical data bank for the ultimate strength of steel structures. U. S. Japan Seminar on Inelastic Instability of Steel Structures and Structural Elements, 1981.

(Recieved August 22, 1983) 